\title{
Análisis de las propiedades psicométricas de una escala para medir el deterioro de las relaciones sentimentales en jóvenes del departamento de Puno
}

\author{
Analysis of the psychometric properties of a scale to measure the deterioration of \\ romantic relationships in young people from the department of Puno
}

\author{
Liz Amanqui Apaza', Yesica Quispe Chuquitarqui², Alcides Quispe Mamani ${ }^{3}$
}

\begin{abstract}
RESUMEN
Objetivo: analizar las propiedades psicométricas de una escala para medir el deterioro de las relaciones sentimentales en jóvenes del departamento de Puno. Metodología: diseño no experimental, de corte transversal, tipo instrumental y de enfoque cuantitativo, está conformada por 600 sujetos que participaron voluntariamente del estudio a partir de una aplicación ambulatoria, no se utilizó algún tipo de muestreo, las características de la población muestran que la mayoría perecen a ambos géneros, sus edades son entre 18 a 26 años y todos ellos establecen una relación sentimental. Resultados: la escala "IRTEP", en su versión traducida, posee un índice de validez $(V=.958)$ a partir de la evaluación de cuatro criterios de evaluación (claridad, contexto, congruencia y dominio del constructo) en 6 jueces. Respecto a la validez de contenido, se obtuvo mediante las pruebas de KMO y esfericidad de Bartlett cuyos valores $(\mathrm{KMO}=.942 ; \mathrm{p}<, 05)$ indicarían que el instrumento es apropiado para un análisis factorial confirmatorio (AFC), la cual sugiere que el instrumento debe poseer un contenido distribuido en 4 factores fijos en la extracción, se logró explicar el $47,6 \%$ de la varianza explicada. Razones que llevaron a un análisis más detallado como lo son ecuaciones estructurales. Los hallazgos demostraron un RMSEA =,049 menor del ,05; CFI=,911 y un TLI=,903 ambos mayores del ,90; estos resultados demuestran un adecuado ajuste de modelo. Conclusión: el instrumento evidencia una buena consistencia interna y validez de constructo.
\end{abstract}

Palabras clave: Control; autonomía; agresión; desconfianza; deterioro; toxico; relaciones en jóvenes.

\section{ABSTRACT}

Objective: to analyze the psychometric properties of a scale to measure the deterioration of romantic relationships in young people from the department of Puno. Methodology: non-experimental design, cross-sectional, instrumental type and quantitative approach, it is made up of 600 subjects who voluntarily participated in the study from an outpatient application, some type of sampling was not used, the characteristics of the population show that the most perish to both genders, their ages are between 18 to 26 years and all of them establish a sentimental relationship. Results: the "IRTEP" scale, in its translated version, has a validity index $(V=.958)$ based on the evaluation of four evaluation criteria (clarity, context, congruence and command of the construct) in 6 judges. Regarding content validity, it was obtained using the KMO and Bartlett's sphericity tests, whose values (KMO = .942; $\mathrm{p}$ $<.05)$ would indicate that the instrument is appropriate for a confirmatory factor analysis (CFA), which suggests that The instrument must have a content distributed in 4 fixed factors in the extraction, it was

\footnotetext{
${ }^{1}$ Universidad Peruana Unión, Lima, Perú

Orcid ID: 0000-0002-0671-7331

${ }^{2}$ Universidad Peruana Unión, Lima, Perú

Orcid ID: 0000-0002-6189-6547

3Universidad Peruana Unión, Juliaca, Perú

Orcid ID: 0000-0002-7404-7464
} 
possible to explain $47.6 \%$ of the explained variance. Reasons that led to a more detailed analysis such as structural equations. The findings demonstrated an RMSEA $=.049$ less than $.05 ; \mathrm{CFI}=.911$ and a $\mathrm{TLI}=.903$ both greater than .90 ; these results demonstrate an adequate model fit. Conclusion: the instrument shows good internal consistency and construct validity.

Keywords: Control; autonomy; aggression; distrust; deterioration; toxic; relationships in young people.

\section{INTRODUCCIÓN}

La presente investigación pretende analizar las propiedades psicométricas de una escala de deterioro de las relaciones sentimentales en jóvenes, para ello es la elección de este tema ya que se debe a la inquietud de la problemática , ya que los jóvenes establecen relaciones sentimentales toxicas, por conocer la magnitud que tiene este fenómeno aparentemente común, puesto a que las relaciones de pareja son entendidas como una problemática de análisis, en la que los integrantes de la relación afectiva permanecen la mayor parte del tiempo experimentado sentimientos vividos. De esta manera se aporta información, resaltando que el deterioro de las relaciones sentimentales causa un impacto e inestabilidad emocional negativa entre las dos personas. El objetivo general de la investigación es analizar las propiedades psicométricas de un instrumento para medir el nivel de deterioro en relaciones sentimentales en jóvenes del departamento de Puno, 2021, así mismo los objetivos específicos es determinar el índice de validez y confiabilidad del contenido de la escala.

En el Perú no existe un instrumento que detecte el nivel de deterioro de las relaciones sentimentales que establecen los jóvenes también llamados "relaciones toxicas", aportando así a la visualización de este mal social y siendo útil como un instrumento de investigación que ayudara significativamente a los jóvenes a detectar casos con violencia dentro de la relación sentimental (Banda \& Sarmiento, 2018).

Según Valenzuela y Vega (2015) una relación toxica tiene tres etapas, dando inicio en la manifestación de bromas hirientes, burlas, infidelidades, humillaciones, prohibiciones siendo así un maltrato psicológico y emocional; luego ingresando a la segunda etapa que da referencia a la destrucción de artículos personales y golpes en modo de broma o discusiones siendo una agresión física.
Finalizando en la tercera etapa donde la persona llega a aislar, amenazar, violentar y asesinar a su pareja.

Existe diferentes razones por las cuales una pareja se ve envuelto en una relación deteriorada, estas causas pueden inferir en elementos psicológicos propios de cada sujeto tales como la baja autoestima e inmadurez emocional, el no tener conocimiento de la identidad e individualidad propia y de su pareja, dificultad para comunicarse y comprender al otro, el no tener reglas y limites claras, el acontecimiento de miedos, angustias, celos, envidia, inseguridad y deseos de control o dominio, pues este tipo de relaciones se caracterizan por la mutua dependencia y circularidad, con alianzas inconscientes en las que el estado mental y emocional dan expectativas sobre la relación y la pareja a si mismo llega a convertirse en necesarias, indispensables e insoportables en el sujeto (Sanchez, García \& Luiz, 2018).

La violencia es un hecho social que refleja la crisis de la sociedad. El resultado es la conformación de un hogar violento tanto física como emocionalmente. Esta situación familiar influye negativamente en el proceso de crecimiento de los hijos que confunden sus sentimientos, experimentan las manipulaciones, ataques a la autoestima, insultos y gritos que reproduce un espiral de violencia. Obviamente, esta relación negativa genera un daño físico y emocional. El dominio y el control de la persona amada disminuye la potencialidad afectiva en la medida en que las conductas agresivas ocasionan sufrimientos en la víctima (Mullisaca, 2018).

Cuando en las relaciones de pareja se confunde el enamoramiento con el amor, las personas llegan a justificar el sufrimiento afectivo $y$ terminan enredadas en una relación negativa ya que erróneamente creen que el amor es así y hay que hacer todo por él; en estas relaciones los individuos se ven sometidos a un gran desgaste emocional y muchos de ellos se 
debilitan psicológicamente, dejando de lado su propia personalidad y esencia como individuos llegando hasta el punto que se deteriora la relación (Álvarez \& García, 2017).

El Centro de Emergencia Mujer (CEM, 2018) revelan que el Perú posee un problema latente respecto a violencia psicológica y física, tanto para varones y mujeres que están establecen relaciones sentimentales, y estas estadísticas nos indican que en enero se reportaron alrededor de 18466 casos nuevos de los cuales 15855 son mujeres y 2611 , varones. Los casos atendidos según el grupo de edad revelan que las personas de 15 a 23 años poseen un total de 2229 casos siendo el tercer grupo de riesgo solo superado por personas de 25 a 30 años.

Para Cojitambo (2017) revela que existe un porcentaje de mujeres que han sufrido violencia por sus parejas, determinando que la forma de agresión más frecuente es la psicológica, con el $43,4 \%$ según el análisis de los resultados estadísticos de una encuesta nacional sobre las relaciones familiares y violencia de género contra las mujeres realizado en Quito en por el Instituto Nacional de Estadísticas y Censos (INEC) y el Consejo Nacional para la Igualdad de Género. Se conoce más a fondo acerca de este problema social, se aportó a nivel individual, familiar y social, además que es un avance para la actividad profesional de futuros Orientadores Familiares.

En el hallazgo de su estudio Diaz (2020) tuvo como objetivo determinar la relación entre la violencia de pareja y dependencia emocional en estudiantes de una Universidad Privada de Trujillo. El diseño utilizado fue diseño no experimental, correlacional y transversal. La muestra estuvo conformada por 166 estudiantes de ambos sexos de entre 15 a 19 años de los primeros ciclos. Se utilizó el Inventario de Violencia en las Relaciones de pareja (CADRI) y el Cuestionario de Dependencia Emocional. Los resultados obtenidos en la muestra total indicaron que no se detectó evidencia de correlación significativa de $p>.05$, tanto de la violencia cometida y de sus dimensiones, como de la violencia sufrida y de sus dimensiones, con la Dependencia emocional a nivel general en los estudiantes que formaron parte de la investigación. Por otro lado, se encontró evidencia de correlación significativa, tanto cometida como sufrida, con las dimensiones de la dependencia emocional.
Por otro lado, Rodríguez San Miguel (2018) en su investigación pretende determinar la relación entre sexismo y la violencia en el enamoramiento en estudiantes de la Universidad Católica Santa María, además de determinar el área profesional, el género, la edad y el tiempo de relación de los estudiantes que influyen en estas dos variables. La muestra estuvo conformada por 298 estudiantes del VI semestre de las 4 áreas profesionales: ciencias de la salud (25\%), ciencias e ingenierías (25\%), ciencias jurídicas $(25 \%)$ y ciencias sociales $(25 \%)$. De la muestra $133(44,6 \%)$ fueron hombres y 165 mujeres $(55,4 \%)$. Los instrumentos psicológicos utilizados fueron la Escala de Sexismo General (ESG) y el Cuestionario de Violencia entre Novios (CUVINO). Se encontró una correlación débil positiva de 0.16 entre Sexismo y Violencia en el enamoramiento con valor de significancia de $p<0.05$.

Según refieren Rozo, Moreno, Perdomo \& Avendaño (2019) su presente investigación tuvo como objetivo elaborar un modelo sobre la asociación de los problemas de adaptación con la agresión y la victimización de la violencia en las relaciones de parejas adolescentes colombianos. Se tomó una muestra de 599 adolescentes con edades entre 13 y 19 años, escolarizados en instituciones educativas públicas y privadas de Bogotá, Se aplicó el cuestionario del Sistema de Evaluación de la Conducta de Niños y Adolescentes, versión autoinforme, y el inventario de conflicto en las relaciones en el noviazgo adolescente. Para el análisis de los datos, se utilizó la técnica de ecuaciones estructurales. Se presentan dos modelos determinados diferencialmente por la variable sexo: en los hombres los problemas de adaptación se relacionan con la agresión y en las mujeres se asocian con la victimización, hallazgos que ofrecen lineamientos para la prevención e intervención de la violencia de pareja en adolescentes.

Contemplando estos antecedentes se hace de vital importancia un instrumento para detectar el grado de deterioro para que posteriormente se les pueda brindar ayuda a los jóvenes de acuerdo a las dimensiones afectadas. Las dimensiones que se han utilizado para la creación de este instrumento son factores que dañan las relaciones sentimentales para finalmente convertirla en toxica, dañando así a ambas partes. 
Tabla 1

Conceptos teóricos referentes a los componentes del deterioro en las relaciones de pareja

\begin{tabular}{|c|c|}
\hline Dimensión & Teoría \\
\hline Control: & $\begin{array}{l}\text { Se ejerce el poder en una relación que quiere saberlo todo, cuando en una pareja existe } \\
\text { alguien que rinde cuentas la relación es de poder y asimétrica muy perniciosa, cabe agregar } \\
\text { que el control por lo general se exhibe y genera siempre el malestar de la relación (Gámez, } \\
\text { Borrajo\& Calvete, 2018). }\end{array}$ \\
\hline Autonomía: & $\begin{array}{l}\text { Conservar la autonomía y respetar la de la pareja es parte del éxito en una relación. Re- } \\
\text { cuerde que se trata de la unión de dos personasdiferentes que, de alguna forma, necesitan } \\
\text { su espacio para desarrollarseen plenitud (Chavarría, 2013). }\end{array}$ \\
\hline Agresión: & $\begin{array}{l}\text { La violencia durante el enamoramiento es definida como todo ataqueintencional de tipo se- } \\
\text { xual, físico o psíquico, de un miembro de la pareja contra el otro estableciendo una relación } \\
\text { integrada por jóveneso adolescentes (Pazos, Oliva \& Hernando, 2014). }\end{array}$ \\
\hline Desconfianza: & $\begin{array}{l}\text { La desconfianza en la pareja puede aparecer por diversas razones, la mayor parte de ellas } \\
\text { se debe a las mentiras, los celos o infidelidad enalgún momento de la relación; y cuando } \\
\text { esta última es descubierta, lapersona engañada perderá la confianza en su pareja. La con- } \\
\text { fianza es el pilar fundamental de la vida en común (Núñez, Canto \& Seebach, 2015). }\end{array}$ \\
\hline
\end{tabular}

\section{METODOLOGÍA}

En este presente estudio de investigación se optó por un diseño no experimental, puesto que el objetivo no es la modificación de la variable; es de tipo de estudio instrumental por que pretende estudiar las propiedades psicométricas de un cuestionario que mide el deterioro de las relaciones sentimentales. Es de enfoque cuantitativo por que el instrumento usado proporciona resultados numéricos las cuales serán procesador bajo esta índole y de corte transversal por que se aplicara el instrumento en un solo punto de tiempo (Hernández, Fernández \& Baptista, 2014).

\section{Participantes}

Respecto a la población considerara en el estudio, se contó con jóvenes que oscilen entre 18 a 26 años, todos procedentes del departamento de Puno; estas personas establecen una relación sentimental, Esta población alcanza los 600 sujetos. La muestra es te tipo no probabilístico, ya que trabajamos en bases a criterios de inclusión y de exclusión.

Los criterios de inclusión que se considera en el estudio:

- Personas que tengan edades de 18 a 26 años.

- Personas de ambos sexos.

- Personas que accedan participar del estudio.

- Personas que tengan una relación sentimental.

\section{Instrumentos}

El instrumento utilizado es el cuestionario de Deterioro de las Relaciones Sentimentales "IRTEP" cuya autoría es propia de Amanqui y Leon, fue creado en el año 2019, posee 39 ítems destinados a identificar el nivel del deterioro de las relaciones sentimentales que establecen los jóvenes, estos ítems están distribuidos en 4 dimensiones (Control, Autonomía, Agresión \& Desconfianza). Los valores de validez fluctúan en $0.8 \mathrm{y}$

0.9 por dimensiones, y su grado de confiabilidad, entre 0.8 y 0,9 ; lo que indica que el cuestionario es confiable y válido para el contexto muestral (Amanqui-Apaza \& Leon-Nureña, 2020).

\section{Procesamiento de datos}

En base a la teoría, se formuló una matriz instrumental de la variable, a partir el constructo del cuestionario de deterioro de las relaciones sentimentales "IRTEP". El instrumento en su versión final, fue revisado por 6 jueces expertos, a partir de ellos que identificaron los índices de validez de contenido por $\mathrm{V}$ de Aiken. Para determinar la validez de constructo, se utilizó el análisis factorial exploratorio, bajo el método de máxima verosimilitud, además, se obtiene la tabla de varianza total explicada la cual justifica la existencia de un determinado número de factores, aunado a ello, se extrajo la matriz de componente rotado, para determinar la pertinencia de los ítems por cada factor 
considerado la carga factorial, y finalmente se generó una tabla percentilar, con tres puntos de corte (alto, medio y bajo) para posibles interpretaciones del instrumento.

\section{RESULTADOS}

\section{Validez de contenido}

En la tabla 2 se observan los índices de acuerdo bajo la fórmula $V$ de Aiken con cuatro criterios de validez de contenido (Claridad, congruencia, contexto y dominio del constructo) a partir de la revisión del contenido en 6 jueces expertos. Los valores de $\mathrm{V}$ de Aiken demuestran que en ningún criterio de validez se obtienen puntuaciones menores al 0,8; por tanto, el instrumento cumple con criterios de validez; los índices de acuerdo demuestran que la dimensión control alcanzan un grado de validez de $V=, 958$; de la misma manera autonomía un $V=, 975$; Agresión un $\mathrm{V}=, 988$; Desconfianza un $\mathrm{V}=, 975$ y finalmente un $\mathrm{V}=, 974$ en la escala general.

Tabla 2

Validez de contenido de la prueba por $V$ de Aiken por dimensiones

\begin{tabular}{llllll} 
Dimensiones & Claridad & Congruencia & Contexto & $\begin{array}{l}\text { Dominio de } \\
\text { constructo }\end{array}$ \\
Control & 0.987 & 0.950 & 0.933 & 0.967 & 0.958 \\
Autonomía & 1 & 0.950 & 1 & 0.950 & 0.975 \\
Agresión & 0.950 & 1 & 1 & 1 & 0.988 \\
Desconfianza & 0.950 & 1 & 0.950 & 1 & 0.975 \\
Total & 0.971 & 0.9975 & 0.971 & 0.979 & 0.974 \\
\hline
\end{tabular}

Nota: La validez obtenida se procesó por $\mathrm{V}$ de Aiken mediante el juicio de 6 expertos (todos mínimamente habilitados por el Colegio de Psicólogos del Perú y uno de ellos, con grado académico de Maestro en Educación con mención en investigación y docencia universitaria

\section{Validez de constructo}

Para hallar un modelo ajustado se ejecutaron análisis factorial confirmatorio bajo el método de máxima verosimilitud con medidas de rotación Varimax. Estos resultados demostraron un $\mathrm{KMO}=, 942$ y una prueba de esfericidad de Bartlett $p<, 05$; estos datos infieren que el modelo tiene un mejor ajuste a partir de la intercorrelación de los reactivos. Al considerar 4 factores fijos en la extracción, se logró explicar el $47,6 \%$ de la varianza explicada. Razones que llevaron a un análisis más detallado realizando las ecuaciones estructurales. Los hallazgos demostraron un RMSEA =,049 menor del ,05; $\mathrm{CFI}=, 911$ y un TLI=,903 ambos mayores del
,90; estos resultados demuestran un adecuado ajuste de modelo. En la figura 1, se observan las variables observadas (ítems) cuya carga factorial permiten agruparlas en variables latentes (dimensiones). Las covarianzas demuestran índices menores de 0,8, por tanto, aseveramos que las variables latentes son diferentes entre sí pero que igualmente aportan a la explicación de la varianza. Por otro lado, los reactivos demuestran una carga factorial no menor al 0,5. 
Tabla 3

Prueba de KMO y esfericidad de Bartlett

\section{Prueba de KMO y Bartlett}

$\begin{array}{lll}\text { Medida Kaiser-Meyer-Olkin de adecuación de muestreo } & .942 \\ \text { Prueba de esfericidad de Bartlett } & \text { Aprox. Chi-cuadradoGI } & 8154.342 \\ & \text { Sig. } & 561 \\ \end{array}$

Figura 1.

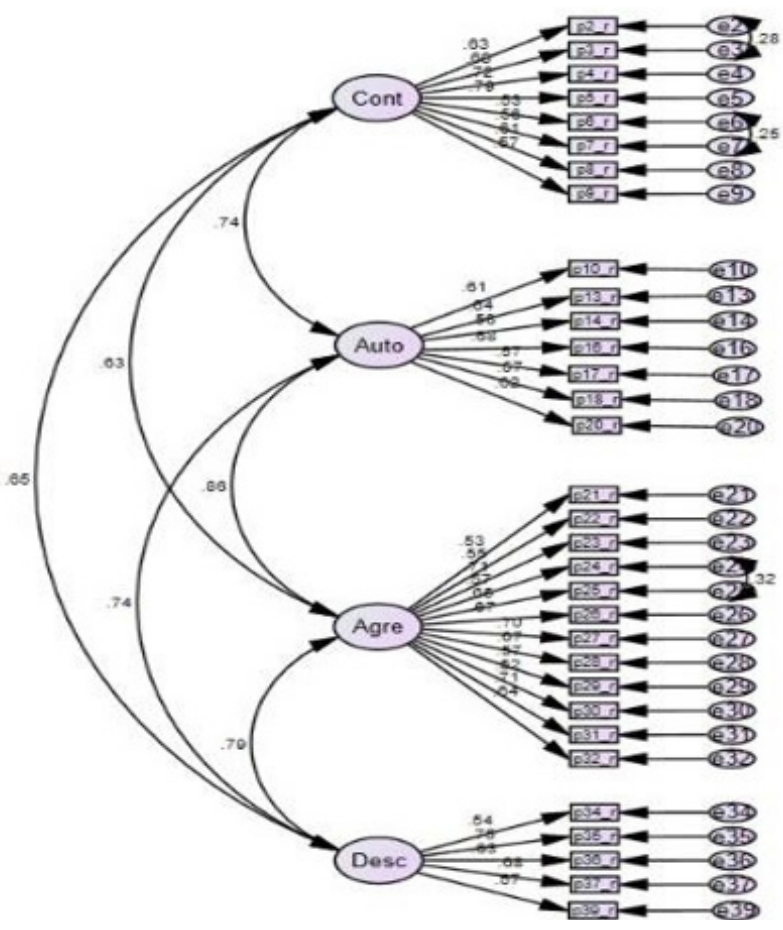

Modelo empírico de la variable

\section{Confiabilidad}

En la tabla 4, se observan los índices de consistencia interna pura a partir de los ítems que menor se intercorrelaciona en el modelo de ecuaciones estructurales. Se observa que la dimensión control alcanza un $\alpha=, 851$; la dimensión autonomía logra un $\alpha=, 819$; la dimensión agresión un $\alpha=, 883$; desconfianza un $\alpha=, 767$; y finalmente la escala general un $\alpha=, 937$. Estos resultados no mayores de 0,8 , por tanto, se cumplen la propiedad psicométrica de confianza instrumental.

Tabla 4

Alfa de Cronbach de la escala de deterioro de las relaciones sentimentales

\begin{tabular}{llll} 
Variable & Ítems & Alfa de Cronbach & Nivel de confianza \\
Deterioro de relacionessentimentales & 34 & 0,937 & Alta confiabilidad \\
Control & 8 & 0,851 & Alta confiabilidad \\
Autonomía & 9 & 0,819 & Alta confiabilidad \\
Agresión & 12 & 0,883 & Alta confiabilidad \\
Desconfianza & 5 & 0,767 & Confiable \\
\hline
\end{tabular}




\section{Baremación}

En la tabla 5 se observa la baremación la cual proporciona una orientación numérica respecto a la interpretación de las puntuaciones obtenidas del instrumento.

Tabla 5

Baremación estandarizada

\begin{tabular}{lllllll} 
PC & Control & Autonomía & Agresión & Desconfianza & $\begin{array}{l}\text { Deterioro de relaciones } \\
\text { sentimentales }\end{array}$ & Nivel \\
\hline $5-25$ & $1-16$ & $1-18$ & $1-22$ & $1-10$ & $1-67$ & Bajo \\
$30-70$ & $17-19$ & $19-22$ & $23-27$ & $11-12$ & $68-80$ & Medio \\
$75-99$ & 20 a mas & 23 a mas & 28 a mas & 13 a mas & 81 a mas & Alto \\
Media & 17,5 & 20,0 & 24,8 & 10,6 & 73,0 & 12,2 \\
D.E & 3,6 & 3,5 & 4,9 & 2,2 & $1-34$ & \\
Ítems & $1-8$ & $9-17$ & $18-29$ & $30-34$ & & \\
\hline
\end{tabular}

\section{DISCUSIÓN}

El objetivo general de la investigación es analizar las propiedades psicométricas de un instrumento para medir el nivel de deterioro en relaciones sentimentales en jóvenes del departamento de Puno. A partir del procedimiento estadístico se encontró que existe diferencias significativas en las dimensiones de control, autonomía, agresión, desconfianza, generando a si un grado de validez de $97 \%$ por la fórmula de $\checkmark$ de Aiken, así mismo estos resultados del análisis factorial demostraron un $\mathrm{KMO}=, 942$ y una prueba de Esfericidad de Bartlett de $p<, 05$; Estos resultados guardan relación con (Rodriguez y Aranda, 2020) el presente estudio tuvo como objetivo determinar las diferencias en los síntomas psicopatológicos entre mujeres víctimas y no víctimas de violencia del centro poblado Alto Trujillo. Es de tipo transversal, con un diseño descriptivo comparativo con un muestreo no probabilístico. La muestra estuvo conformada por 309 mujeres, 155 víctimas y 154 no víctimas de violencia. Para obtener los datos, se utilizó la ficha de tamizaje VIF, creado por el Ministerio de salud y el SCL90-R creado por Derogatis. Los resultados obtenidos entre víctimas y no víctimas de violencia indicaron diferencias altamente significativas en las 9 dimensiones y los 3 índices globales, con un tamaño de efecto mediano. Los resultados de la presente investigación no guardan relación a los propuesto por (Sanchez, García \& Luiz De Andrade, 2018) hay diferentes razones por las cuales una pareja se ve involucrado en una relación deteriorada, estas causas pueden derivarse de elementos psicológicos propios de cada sujeto tales como la inmadurez emocional y baja autoestima, el no tener conocimiento de la identidad e individualidad propia y de su pareja, dificultad para comunicarse y comprender al otro, el no tener reglas y limites claras, el acontecimiento de miedos, angustias, celos, envidia, inseguridad y deseos de control o dominio, pues este tipo de relaciones se caracterizan por la mutua dependencia y circularidad, con alianzas inconscientes en las que el estado mental y emocional se ven afectados. Algunos autores respaldan los resultados como Fernández (2020) menciona determinar la relación entre violencia de pareja o dependencia emocional en jóvenes.

\section{Declaración de financiamiento y de conflictos de interés:}

El estudio fue financiado por los autores, quienes declaran no tener conflictos de interés

\section{Correspondencia}

\section{Liz Amanqui Apaza}

Correo electrónico:

liz.amanqui@upeu.edu.pe

\section{Yesica Quispe Chuquitarqui}

Correo electrónico:

yesica.qc@upeu.edu.pe

\section{Alcides Quispe Mamani}

Correo electrónico:

alcides.quispe@upeu.edu.pe 


\section{REFERENCIAS}

Álvarez Ramirez, E., \& García Méndez , M. (2017). Estilos de amor y culpa como predictores de la satisfacción marital en hombres y mujeres. Revista Cientifica de América Latina, 76-85. https://www.redalyc.org/pdf/292/29251161007. pdf

Amanqui Apaza, L. F., \& Leon Nureña, M. E. (2020). Construcción y análisis de las propiedades psicométricas de una escala de deterioro de relaciones sentimnetales "IRTEP" en jóvenes de la ciudad de Juliaca, año 2019. Repositorio de la Universidad Peruana Unión, Juliaca, PeruPuno. https://repositorio.upeu.edu.pe/bitstream/ handle/UPEU/3636/Liz_Trabajo_Bachiller_2020. pdf?sequ ence $=1 \&$ isAllowed $=y$

Banda Andrade, M. R., \& Sarmiento Leyra, G. Y. (2018). Autoestima y dependencia emocional, en jóvenes víctimas y no víctimas de violencia en la etapa de enamoramiento Institutos de Educación Pública - Arequipa. Repositorio Institucional de la UNSA. http://repositorio.unsa.edu.pe/handle/ UNSA/6527

Centro de Emergencia Mujer, C. (2018). Informe Estadistico, Programa Nacional contra la Violencia Familiar y sexual. Ministerio de la Mujer y Poblaciones Vulnerables. https://www. mimp.gob.pe/files/programas nacionales/ pncvfs/publicaciones/informe-estadistico02_2018-PNCVFS-UGIGC.pdf

Chavarría Arango, Z. P. (2013). Autonoma Emocional. Medellin. https://repository.ces. edu.co/bitstream/10946/863/2/PROYECTO_ INTERVENCION_AUTOMONI A_EMOCIONAL. pdf

Cojitambo Morocho, J. J. (2017). la violencia psicológica en el enamoramiento adolescente [tesis de licenciatura, Universidad de Ecuador]. Cuenca - Ecuador. https://dspace.ucuenca.edu. ec/bitstream/123456789/27722/1/TESIS\%20 PDF..pdf

Diaz Fernández, R. A. (2020). Violencia de pareja y dependencia emocional en estudiantes de una Universidad Privada de trujillo [tesis de Doctorado, trujillo]. UCV-Institucional, TrujilloPeru. https://repositorio.ucv.edu.pe/bitstream/ handle/20.500.12692/48461/D\%c3\%adaz FRASD.pdf?sequence=1\&isAllowed=y

Gámez Guadix, M., Borrajo, E., \& Calvete, E. (2018). Abuso, control y violencia en la pareja. Obtenido de https://www.redalyc.org/ jatsRepo/778/77857281013/html/index.html

Hernández Sampieri, R., Fernández Collado, C., \& Baptista Lucio, P. (2014). Metodología de la Investigación. Sexta edición. https://www.uca.ac.cr/ wp- content/uploads/2017/10/Investigacion.pdf
Mullisaca Jaen, D. N. (2018). Vivencias de violencia en el proceso del enamoramiento de los jóvenes de la Universidad Nacional de Juliaca -2017. Repositorio Institucional UNA - PUNO, Puno Perú. http://repositorio.unap.edu.pe/bitstream/ handle/UNAP/10528/Mullisaca_Jaen_Deisy_ Nelida.pdf?se quence=1\&isAllowed=y

Núñez, F., Canto Mila, N., \& Seebach, S. (2015). Confianza, mentira y traición el pael de la confianza y sus sombras en las relaciones de pareja.http://www.scielo.org.mx/pdf/soc/v30n84/ v30n84a4.pdf

Pazos Gómez, M., Oliva Delgado, A., \& Hernando Gómez, Á. (s.f.). Violencia en adolescente. Revista Latinoamerica de Psicología. https:// www.redalyc.org/pdf/805/80533065002.pdf

Rodríguez San Miguel, D. (2018). Sexismo y violencia en el enamoramiento en estudiantes Universitarios.Repositorio Institucional UCSM- Tesis, Arequipa. http://tesis.ucsm. edu.pe/repositorio/bitstream/handle/ UCSM/8473/76.0375.PS.pdf?sequence=1\&isA llowed=y

Rozo Sánchez, M. M., Moreno Mendez, J. H., Perdomo Escobar , S. J., \& Avendaño Prieto, B. L. (2019). Modelo de violencia en relaciones de pareja en adolescentes colombianos. Suma Psicológica, 55-. http://www.scielo.org.co/pdf/ sumps/v26n1/0121-4381-sumps-26-01-55.pdf Sanchez

Aragón, R., García Meraz, M., \& Luiz De Andrade, A. (2018). táctica de resolución de conflicto en las relaciones de pareja: validación en dos ciudades de México y una en Brasil. Universidad Nacional Autonoma de Mexico. https://www.medigraphic. com/pdfs/revmexinvpsi/mip-2018/mip181e.pdf

Sánchez, E. (2020). Dos factores clave en el deterioro de la relación de pareja. . La mente es maravillosa. https://lamenteesmaravillosa.com/dos-factoresclave-en-el-deterioro-de-la-relacion-de- pareja/

Valenzuela Varela, A., \& Vega López, M. G. (2015). Violencia en el noviazgo en adolescentes un problema de salud pública. Universidad de Guadalajara, Centro Universitario de Ciencias de la Salud. https://www.medigraphic.com/pdfs/ saljalisco/sj-2015/sj153g.pdf

Recibido: 16/07/2021

Aceptado: 19/11/2021 